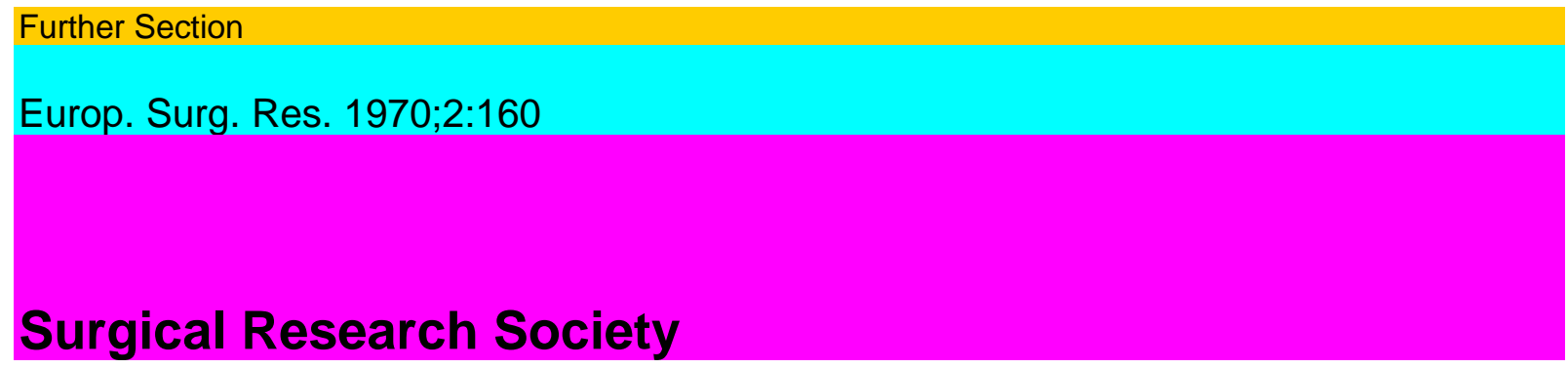

\title{
Meetings 1970/71
}

2nd to 4th July 1970

Location: Cambridge

Closing date for Abstracts: Monday, 13th April 1970

8th to 9th January 1971

Location: London

Closing date for Abstracts: Monday, 26th October 1970

Abstracts to be submitted to: Professor H. L. Duthie, University Department of Surgery, The Royal Infirmary, Sheffield 6, Yorkshire, England, from whom any further information may be obtained.

\section{Preparation of Summaries}

Summer Meeting, 1970

The summaries of papers being presented at this meeting will appear later, as usual, in the British Journal of Surgery. To help in the task of preparing these summaries for the programme and for publication, members submitting summaries are requested to ensure that they conform to the following requirements:

1. Seven copies of each summary should be submitted. The text must not exceed 200 words in length, and should be typed in double spacing, on one quarto sheet.

2. The title of the paper should accompany the text on every copy.

3. On the top copy only, the authors' surname and initials should be given (in the case of women one of their Christian names should be set out in full), but not titles or degrees. In cases of joint authorship, the name of the person who will read the paper must be placed first. If none of the authors is a member of the Society, the name of the member introducing the paper must be added in brackets after the name of the author(s). Also on the top copy only, the Hospital or School of origin should be stated.

4. It is essential that summaries should be suitable for publication as, in the past, much editorial work has been needed to make the summaries suitable for publication in the British Journal of Surgery. This means that data must be included, and results and conclusions must be clearly described. Phrases like 'the results of these studies will be described and discussed' are not acceptable. Once a paper is accepted, if the authors wish to revise the abstract, the copy for publication must be given to the secretary at the meeting, when the paper is presented.

5. References should be kept to a minimum, and must not exceed four in number. In the case of papers by more than two authors, in the text, only the name of the first author should be set out, followed by the expression $1 / 81$ al. 1 together with the year of publication. At the foot of the summary, the references should be set out in alphabetical order, each one giving, in order, the name and initials of the author(s), year of publication (in brackets), the name of the journal (abbreviated according to the World List of Scientific Periodicals), the volume number (underlined), and the number of the first page of the article. For books, in addition to the author(s), the title, the year of publication, the place of publication and publisher's name must be given.

6. Members introducing papers are responsible for ensuring that the abstracts conform to these requirements.

7. Submissions of summaries that do not conform to these requirements may result in the rejection of papers that are otherwise suitable for this meeting. 